\title{
Orthogonality Catastrophe as a Consequence of the Quantum Speed Limit
}

\author{
Thomás Fogarty $\odot,{ }^{1, *}$ Sebastian Deffner $\odot^{2, \dagger}$ Thomas Busch $\oplus^{1, \$}$ and Steve Campbell $\odot^{3, \S}$ \\ ${ }^{1}$ Quantum Systems Unit, Okinawa Institute of Science and Technology Graduate University, Onna, Okinawa 904-0495, Japan \\ ${ }^{2}$ Department of Physics, University of Maryland, Baltimore County, Baltimore, Maryland 21250, USA \\ ${ }^{3}$ School of Physics, University College Dublin, Belfield Dublin 4, Ireland
}

(Received 25 October 2019; accepted 18 February 2020; published 16 March 2020)

\begin{abstract}
A remarkable feature of quantum many-body systems is the orthogonality catastrophe that describes their extensively growing sensitivity to local perturbations and plays an important role in condensed matter physics. Here we show that the dynamics of the orthogonality catastrophe can be fully characterized by the quantum speed limit and, more specifically, that any quenched quantum many-body system, whose variance in ground state energy scales with the system size, exhibits the orthogonality catastrophe. Our rigorous findings are demonstrated by two paradigmatic classes of many-body systems - the trapped Fermi gas and the long-range interacting Lipkin-Meshkov-Glick spin model.
\end{abstract}

DOI: 10.1103/PhysRevLett.124.110601

Introduction.-Numerous many-body systems exhibit properties and phases that cannot be explained in exclusively classical terms. Famous examples include Bose-Einstein condensation [1,2], topological states [3,4], nonclassical dispersion relations [5,6], and many-body localization [7], to name just a few. Whereas static properties are often understood in great detail, understanding dynamical properties can be significantly more involved. Nevertheless, it is the dynamical properties that are particularly interesting for quantum technological applications, as exemplified by quantum thermodynamic devices [8].

Mathematically, the issue is due to the fact that, to describe the dynamics of many-body systems, the timedependent Schrödinger equation has to be solved for an immense number of microscopic variables, which is practically unfeasible. One way forward is then to obtain qualitative insights from fundamental statements of quantum physics, which, in part, is why the study of the quantum speed limit (QSL) has spurred an area of research in its own right [9]. The QSL is a careful formulation of Heisenberg's uncertainty relation for energy and time [10] and bounds the minimal time, referred to as the QSL time $\tau_{\mathrm{QSL}}$, that a quantum system needs to evolve between distinct states [11-15]. Originally formulated for undriven Schrödinger dynamics [11,13-15], the QSL has been generalized to controlled [16-22] and open systems [23-31].

Interestingly, in its original inception the QSL was formulated to bound the minimal time for the evolution between two orthogonal states [11,14]. It is therefore interesting to consider its relation to the discovery by Anderson [32] that a local perturbation on a gas of $N$ fermions causes a change in the quantum many-body states that is strongly dependent on $N$. In particular, in the limit of large $N$ the local perturbation forces the system to assume an orthogonal state - an effect known as orthogonality catastrophe (OC). The $\mathrm{OC}$ has been analyzed in many different scenarios, including quantum spin models [33,34], trapped gases [35-40], and impurity models [41], and has also been explored in thermal states [42], and in understanding the breakdown of quantum adiabaticity [43]. However, to date, a clear connection between the dynamics as characterized by the QSL and the orthogonality catastrophe has not been made.

In this Letter, we aim at closing this gap in the fundamental understanding of the dynamics of quantum many-body systems. Typically the OC is characterized by the dynamical overlap $\chi(t)$, which is closely related to the Loschmidt echo $[44,45]$ and is defined as the inner product of the state in the absence and presence of the perturbation. We show that the QSL, i.e., the maximal rate with which any quantum many-body system can evolve, is also governed by $\chi(t)$. With this fundamental relation at hand, we then conclude that the OC appears in any quantum many-body system in which the variance of the energy scales with the number of particles $N^{\alpha}$, where $\alpha$ is an exponent determined by the specific system properties. This conclusion is then explored and demonstrated for two important many-body systems: the trapped Fermi gas and the isotropic Lipkin-Meshkov-Glick model [46], which is a paradigmatic example of strongly interacting systems [47-55].

Anderson's orthogonality catastrophe.-In his original formulation, Anderson considered the effect a local perturbation has on a gas of $N$ spinless fermions [32] and showed that the overlap between the perturbed and unperturbed many-body states, written as

$$
\chi=\left\langle\Psi\left(x_{1}, x_{2}, \ldots, x_{N}\right) \mid \Phi\left(x_{1}, x_{2}, \ldots, x_{N}\right)\right\rangle,
$$


scales as $\chi \propto N^{-\alpha / 2}$, where $\alpha$ is related to the perturbation strength. As a consequence, even a small perturbation causes two many-body states to become orthogonal as $N$ grows. Although Anderson's treatment focused on stationary states, dynamical orthogonality after sudden quenches can be similarly observed and is described by a dynamical overlap

$$
\chi(t)=\left\langle\Psi\left|e^{i \mathcal{H}_{f} t} e^{-i \mathcal{H}_{i} t}\right| \Psi\right\rangle,
$$

with the initial state $\Psi$ being an eigenstate of the Hamiltonian $\mathcal{H}_{i}$, while $\mathcal{H}_{f}$ is the perturbed Hamiltonian [56]. This is related to the survival probability or timedependent fidelity $\mathcal{F}(t)=|\chi(t)|^{2}$, which is an important quantifier of out-of-equilibrium dynamics [57-63]. Indeed, one can find footprints of the dynamical OC in the spectral function $S(\omega)=2 \operatorname{Re}\left(\int_{-\infty}^{\infty} d t \chi(t) e^{i \omega t}\right)$, which is broadened by the OC and possesses a power-law tail [64]. While the $\mathrm{OC}$ is well known in condensed matter physics, theoretical studies have proposed using cold atomic systems to observe and study it, due to the ability to create clean many-body states with separately controllable impurity atoms [35,42]. Recent experiments have been able to measure the survival probability and spectral function of a Fermi gas of ${ }^{6} \mathrm{Li}$ after an interaction quench with ${ }^{40} \mathrm{~K}$ impurities by using a Ramsey atom-interferometric technique heralding the OC $[65,66]$.

"Catastrophic" quantum speed limit.-To establish a relation between the OC and the QSL we start by inspecting the dynamical overlap $\chi(t)$. Since $|\Psi\rangle$ is an eigenstate of the unperturbed Hamiltonian $\mathcal{H}_{i}|\Psi\rangle=\mathcal{E}_{i}|\Psi\rangle$, we can write

$$
\chi(t)=\left\langle\Psi\left|e^{i \mathcal{H}_{f} t}\right| \Psi\right\rangle e^{-i \mathcal{E}_{i} t} .
$$

This allows us to introduce the Bures angle between the two states $\left|\psi_{0}\right\rangle=|\Psi\rangle$ and $\left|\psi_{t}\right\rangle=e^{i \mathcal{H}_{f} t}|\Psi\rangle$,

$$
\mathcal{L}(t) \equiv \arccos |\chi(t)|=\arccos \left|\left\langle\psi_{0} \mid \psi_{t}\right\rangle\right|,
$$

which is only implicitly dependent on the unperturbed Hamiltonian $\mathcal{H}_{i}$. At any time $\tau$, the Bures angle has an upper bound given by $[24,67]$

$$
\mathcal{L}(\tau) \leq \frac{1}{2} \int_{0}^{\tau} d t \sqrt{\mathcal{I}}
$$

where $\mathcal{I}$ is the quantum Fisher information with respect to time. For pure states and Hamiltonian dynamics, it can be computed explicitly as [68]

$$
\mathcal{I}=4\left(\left\langle\mathcal{H}_{f}^{2}\right\rangle-\left\langle\mathcal{H}_{f}\right\rangle^{2}\right)=4 \Delta \mathcal{H}_{f}^{2},
$$

and one can therefore immediately see that the dynamics, when described by the dynamical overlap, is fully characterized by the variance of the perturbed Hamiltonian $\mathcal{H}_{f}$.
Introducing now the well-known connection between the QSL and the quantum Fisher information, $v_{\mathrm{QSL}} \equiv \sqrt{\mathcal{I}} / 2$ $[9,24,30,69,70]$, one can see that the QSL can be written as $v_{\mathrm{QSL}}=\Delta \mathcal{H}_{f}$. Resubstituting this into Eq. (5), and noting that $\Delta \mathcal{H}_{f}$ is time independent, then gives a direct connection between the QSL time and the dynamical overlap as

$$
\tau \geq \tau_{\mathrm{QSL}}=\frac{\arccos |\chi(\tau)|}{\Delta \mathcal{H}_{f}}
$$

The maximal rate of quantum evolution $v_{\mathrm{QSL}}$ is therefore determined by the energy variance of the perturbed Hamiltonian, which is a function of the number of particles $N$. As a consequence, we see that $\tau_{\mathrm{QSL}} \rightarrow 0$ when $\Delta \mathcal{H}_{f}$ scales extensively with $N$, which means that the time a large system needs to evolve between two orthogonal states vanishes. We then see that the $\mathrm{OC}$ is a consequence of the quantum speed limit: an extensive postquench Hamiltonian variance drives the many-body system to evolve significantly faster, and correspondingly, the time to reach any orthogonal state vanishes.

Orthogonality catastrophe and other QSLS.-It is worth noting that $\chi(t)$ as given in Eq. (2) is closely related to the thermodynamic work $W$ performed in perturbing the manybody system. Thus far, by virtue of the sudden quench approximation, it is clear that for $t \geq 0^{+}$the Hamiltonian is time independent and the ensuing dynamics unitary. As such, it is easy to convince oneself that the MandelstamTamm bound, virtually in its original form, presents a natural choice for exploring the OC. However, this picture does not explicitly account for the switching on of the interaction, which necessarily requires some work to be performed $[39,55,71]$. We can explore this connection in a concrete manner by exploiting the fact that QSL times can be derived for any given distinguishability metric [30]. Choosing Eq. (2) as our figure of merit, we can derive an alternative expression for $\tau_{\mathrm{QSL}}$ that carries additional physical significance in terms of the work done in quenching the system $[72,73]$ and find

$$
\tau_{W}=\frac{\hbar\left(1-\left|\chi_{\tau}\right|\right)}{|\langle W\rangle|},
$$

where $\langle W\rangle=\left.\partial_{t} \chi(t)\right|_{t=0}$ is the average work performed due to the quench [73-75] and exhibits similar scaling to $\tau_{\mathrm{QSL}}$. It is worth noting that Eqs. (7) and (8) also demonstrate that the formalism of the QSL provides a useful framework to explore fundamental properties of any given dynamics. As the QSL is inherently dependent on which distinguishability metric is employed, closely related bounds could be derived that account for other features of the system, such as the coherence (see, e.g., Ref. [9] for an overview). By choosing the survival probability, we have established a 
strict relationship between the emergence of the OC and the dynamics and thermodynamics of the quench process.

Trapped Fermi gas.-As a first example, we now explore the above connection in a harmonically trapped Fermi gas, which is close to Anderson's original setting [32]. The $N$-body wave function can be constructed through the Slater determinant of the respective single particle eigenstates

$$
\Psi\left(x_{1}, x_{2}, \ldots, x_{N}\right)=\frac{1}{\sqrt{N !}} \underset{n, j=1}{N}\left[\psi_{n}\left(x_{j}\right)\right],
$$

which are in turn defined before and after a sudden quench by $\mathcal{H}_{i} \psi_{n}=E_{n} \psi_{n}$ and $\mathcal{H}_{f} \phi_{n}=E_{n}^{\prime} \phi_{n}$, respectively. The survival probability of the many-body state is then

$$
\begin{aligned}
\mathcal{F}(t) & =\left|\left\langle\Psi\left|e^{i \mathcal{H}_{f} t} e^{-i \mathcal{H}_{i} t}\right| \Psi\right\rangle\right|^{2} \\
& =|\operatorname{det}[\mathcal{A}(t)]|^{2},
\end{aligned}
$$

where the elements of the matrix $\mathcal{A}$ are the overlaps of the single particle states $\psi_{k}(x, 0)$ and $\psi_{l}(x, t)$ as [32]

$$
\begin{aligned}
\mathcal{A}_{k, l}(t) & =\int_{-\infty}^{\infty} \psi_{k}(x, 0) \psi_{l}^{*}(x, t) d x \\
& =\sum_{m=1}^{\infty}\left\langle\psi_{k} \mid \phi_{m}\right\rangle\left\langle\psi_{l} \mid \phi_{m}\right\rangle e^{-i\left(E_{m}^{\prime}-E_{k}\right) t} .
\end{aligned}
$$

This significantly simplifies the calculation of $\chi(t)$ and allows one to consider large systems. Indeed, for a sudden quench in the trapping frequency $\omega_{1} \rightarrow \omega_{2}$ such that $\eta=\omega_{2} / \omega_{1}>1$, the single particle overlaps are known analytically $[59,76]$. The static (i.e., overlap with the ground state) and dynamical survival probabilities can be calculated as

$$
\begin{gathered}
\mathcal{F}=|\langle\Psi \mid \Phi\rangle|^{2}=\left(\frac{2 \sqrt{\eta}}{\eta+1}\right)^{N^{2}}, \\
\mathcal{F}(t)=\left(\frac{2 \eta}{\sqrt{4 \eta^{2} \cos ^{2}(t)+\left(\eta^{2}+1\right)^{2} \sin ^{2}(t)}}\right)^{N^{2}} .
\end{gathered}
$$

One immediately sees that both decay with the exponent $N^{2}$ and depend on the strength of the quench $\eta$. For larger systems, the survival probability decays faster [see inset of Fig. 1(a)], which is the manifestation of the OC.

To determine the QSL time, Eq. (7), we require $\Delta H$ for the Fermi gas, which is given by

$$
\Delta H=\frac{\eta^{2}-1}{2 \sqrt{2} N} \sum_{n=1}^{N} \sqrt{n^{2}-n+1} \approx \frac{N}{4 \sqrt{2}}\left[\eta^{2}-1\right],
$$

where the approximate expression is valid for large particle numbers $N$. The QSL time therefore exhibits an extensive
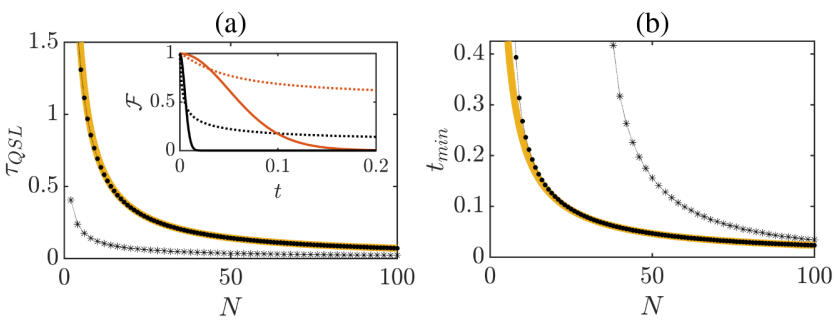

FIG. 1. (a) Dots: QSL time, Eq. (7), as a function of particle number for a trap quench of strength $\eta=1.5$. The yellow line uses the approximate expression for $\Delta H$ in the large $N$ limit. Stars: QSL time for an impurity quench of strength $\kappa=0.5$. (Inset) Survival probability vs time for $N=10$ (red lines) and $N=100$ (black lines) for a trap quench (solid lines) and an impurity quench (dotted lines). (b) Minimum time to reach $\mathcal{F}(t)=10^{-2}$ for the trap quench (dots) with the approximation in Eq. (18) (yellow line), and minimum time to reach $\mathcal{F}(t)=$ 0.25 for the impurity quench (stars).

behavior with the system size [see Fig. 1(a)], which is qualitatively similar to the survival probability. Similarly, the average work is given by $\langle W\rangle=(N / 4)\left[\eta^{2}-1\right]$ and exhibits scaling comparable to $\tau_{\mathrm{QSL}}$ [71]. To formally relate the QSL time and the survival probability, we calculate the minimum time for the latter to reach a specific value, i.e., $\mathcal{F}\left(t_{\min }\right)=\vartheta$, and find from Eq. (15)

$$
t_{\min }=\frac{1}{\pi} \sec ^{-1}\left(\frac{\eta^{2}-1}{\sqrt{1+\eta^{4}+\eta^{2}\left(2-4 \vartheta^{-2 / N^{2}}\right)}}\right),
$$

which for large $N$ reduces to

$$
t_{\min } \approx \frac{2 \eta}{\pi N} \frac{\sqrt{\log \left(\vartheta^{-2}\right)}}{\eta^{2}-1} .
$$

Therefore this minimum time can be related through the energy variance in Eq. (16) to the speed limit as

$$
t_{\min } \sim \tau_{\mathrm{QSL}} \frac{\eta}{\pi^{2}} \sqrt{-\log (\vartheta)},
$$

which shows that the QSL bounds the minimum time to reach $\mathcal{F}\left(\tau_{\mathrm{QSL}}\right)=e^{-\pi^{4} / \eta^{2}}$, as shown in Fig. 1(b). In fact, for sudden quenches, it is not surprising that the appearance of dynamically orthogonality depends on the QSL time, as the variance of the nonequilibrium excitations and the evolution of the survival probability are described by the same distribution of single particle probabilities.

We can also consider the setting first proposed by Anderson, quenching the interaction with an impurity embedded in the Fermi sea, which leads to a power-law decay of the survival probability $[32,35,42]$. Describing the interaction with the impurity as a delta function with a height $N \kappa$, the single particle Hamiltonian can be written as 


$$
H=-\frac{\hbar^{2}}{2 m} \nabla^{2}+\frac{1}{2} m \omega^{2} x^{2}+\Theta(t) N \kappa \delta(x),
$$

where $\Theta(t)$ is the Heaviside step function that suddenly switches on the interaction for $t>0$. Similar to the trap quench, the QSL time and $t_{\min }$ exhibit an extensive dependence on $N$ (see Fig. 1), reaffirming the previous analysis.

Orthogonality catastrophe in interacting systems.-We next consider a more complex setting where an impurity is immersed in an interacting bath. In particular, we choose the model of a single spin interacting with a critical isotropic Lipkin-Meshkov-Glick (LMG) environment $[47,48]$. The total Hamiltonian is given by $H=H_{\mathrm{LMG}}+$ $H_{\text {int }}$ with

$$
\begin{aligned}
H_{\mathrm{LMG}} & =-\frac{\lambda}{N} \sum_{i<j}^{N}\left(\sigma_{x}^{i} \sigma_{x}^{j}+\sigma_{y}^{i} \sigma_{y}^{j}\right)-\sum_{i=1}^{N} \sigma_{z}^{i}, \\
H_{\mathrm{int}} & =\frac{\gamma}{N} \sum_{i}^{N}\left(\sigma_{x}^{i} \sigma_{x}^{s}+\sigma_{y}^{i} \sigma_{y}^{s}\right)-\sigma_{z}^{s} .
\end{aligned}
$$

Here $H_{\text {int }}$ accounts for the impurity-bath interaction term with strength $\gamma$ and the free Hamiltonian of the impurity system $s$. The LMG model is an example of a critical spin system that exhibits a quantum phase transition at $\lambda=1$ [46,49-55]. It is convenient to work in the angular momentum basis in terms of collective spin operators, $S_{\alpha}=\sum_{i}^{N} \sigma_{\alpha}^{i}$. In this picture, the Hamiltonian becomes

$$
\begin{aligned}
H= & -\frac{\lambda}{N}\left(S_{+} S_{-}+S_{-} S_{+}-N \mathbb{1}_{N}\right) \\
& -2 S_{z}-2 \frac{\gamma}{N}\left(s_{+} S_{-}+s_{-} S_{+}\right)-2 s_{z},
\end{aligned}
$$

where we have also used the spin operators for the impurity. In line with the original framework of Anderson, where the impurity corresponded to a small perturbation, and following the previous analysis, we will fix $\gamma=\lambda \sqrt{N}$ such that the impurity interacts comparatively weakly with the bath.

We first examine the behavior of $\mathcal{F}$ for the whole system when the interaction $\gamma$ is suddenly switched on at $t=0$. We initialize both in their respective ground states; i.e., for the impurity, this simply means that it is always initialized in $\left|\psi_{s}\right\rangle=|0\rangle_{s}$, while the ground state of the LMG bath will be dependent on the value of $\lambda$ chosen. For $\lambda<1$ the field dominates and the spins tend to all align, while for $\lambda>1$ the ground state is in the critical phase [47].

Quenching on the interaction, $\gamma=\lambda \sqrt{N}$ drives the system out of equilibrium. In Fig. 2 we examine the survival probability for moderate $N=200$ (solid) and large $N=1000$ (dashed) sized environments for $\lambda=0.9$ and $\lambda=1.1$, Figs. 2(a) and 2(b), respectively, which are representative values for their phases (see Supplemental Material [73]). Clearly, for $\lambda=0.9, \mathcal{F}$ never reaches zero

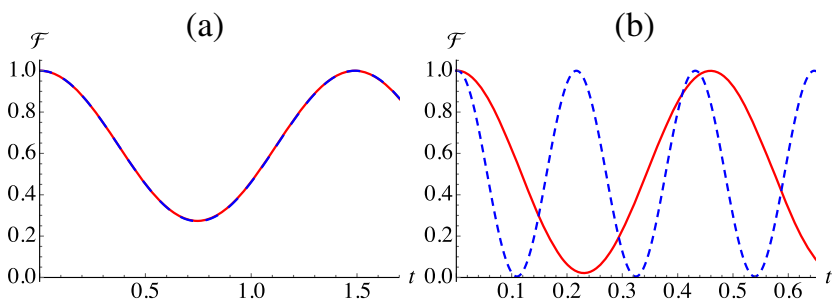

FIG. 2. Survival probability $\mathcal{F}$ of the impurity + environment state when the LMG bath is initialized with (a) $\lambda=0.9$ and (b) $\lambda=1.1$ for different values of total number of environmental spins, $N=200$ (solid, red) and $N=1000$ (dashed, blue).

and, furthermore, its behavior is unaffected by the size of the environment. Therefore, when the LMG model is in this phase, we never witness the OC. In contrast, we clearly see that, for an environment initialized with $\lambda>1$, the overall system periodically evolves to almost orthogonal states for moderate sized environments. As we increase the environmental size, the minimum value of $\mathcal{F}(t) \rightarrow 0$. Thus, for increasing $N$ the evolved state approaches a fully orthogonal state and the time to reach this state is strongly dependent on the size of the bath, as clearly evidenced in Fig. 2(b). These features combined indicate that for $\lambda>1$ the system displays the OC.

In Fig. 3(a) we examine the minimum value of the survival probability $\mathcal{F}_{\min }$ as a function of inverse environment size, $1 / N$ for $\lambda>1$. Each curve from top to bottom corresponds to an increasingly large value of $\lambda \in(1.2,2.0)$. We find a simple linear relationship and it is clear that, as $N \rightarrow \infty, \mathcal{F} \rightarrow 0$ and thus we are witnessing the OC. In contrast, when the spin bath is initialized in the aligned phase, the minimal value of the survival probability is insensitive to the bath size, cf. Fig. 2(a). Figure 3(b) shows the relationship between $\mathcal{F}_{\text {min }}$ and the corresponding time when this minimum occurs, $t_{\min }$. We clearly see that both $\mathcal{F}_{\text {min }}$ and $t_{\min } \rightarrow 0$ as $N \rightarrow \infty$. Thus, Fig. 3 indicates that when the OC manifests it corresponds to a vanishing orthogonality time as the size of the bath is increased, while if the composite system does not reach orthogonality, we find its properties are largely independent of $N$.

We now would like to connect the above features with the QSL time, Eq. (7) and, in particular, shed light onto why despite being a many-body system we do not witness the OC for $\lambda<1$. In general, the energy spectrum of the isotropic LMG model is characterized by a cascade of energy level crossings [73] and we find $\Delta H$ reads

$$
\Delta H=\sqrt{\frac{4(1+j)(N-j) \gamma^{2}}{N^{2}}},
$$

where $0 \leq j \leq N$ indicates how many energy level crossings have occurred. We find a starkly different behavior depending on which phase the LMG spin bath is initialized in. For $0<\lambda<1$, no energy level crossings occur [73], and 
(a)
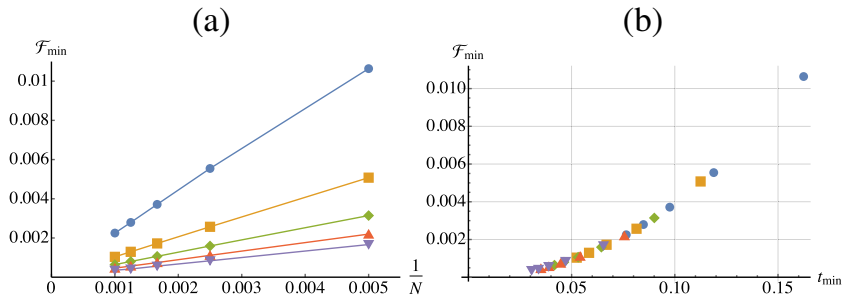

Fund, a donor advised fund of Silicon Valley Community Foundation (S. D.). T. F. and T. B. are supported by the Okinawa Institute of Science and Technology Graduate University. S. C. gratefully acknowledges the Science Foundation Ireland Starting Investigator Research Grant "SpeedDemon" (No. 18/SIRG/5508) for financial support.

FIG. 3. (a) $\mathcal{F}_{\text {min }}$ as a function of $N$. Each line, from top- to bottommost, corresponds to an increasing value of $\lambda \in(1.2,2.0)$ in steps of 0.2 . (b) Minimum value of $\mathcal{F}$ vs corresponding required evolution time $t_{\min }$ for different values of $\lambda=1.2$ (circles), 1.4 (squares), 1.6 (diamonds), 1.8 (up triangles), 2.0 (down triangles). Each consecutive data point approaching the origin corresponds to an increasing value of $N \in(200,1000)$ in steps of 200.

we have $j=0$ and $\Delta H=2 \gamma / \sqrt{N}$. Therefore, since $\gamma=\lambda \sqrt{N}$, it is clear that the variance is independent of the size of the bath in this phase. The fact that we do not see the OC emerging then naturally follows, since regardless of how large the total system is, the QSL time, Eq. (7), is always the same. We find a very different picture emerging for $\lambda>1$, where the energy changes as a function of $\lambda$ due to a cascade of crossings (see Supplemental Material [73]). In particular, as $N$ is increased, the number of energy level crossings occurring becomes increasingly dense. Thus, we find Eq. (23) scales extensively as the environmental size grows. Correspondingly, we have that $\tau_{\mathrm{QSL}} \rightarrow 0$ as $N$ grows, and hence the orthogonality catastrophe follows as a consequence of the vanishing QSL time.

Concluding remarks.-In the present analysis, we achieved several important results. First and foremost, we related the dynamical occurrence of the orthogonality catastrophe with the quantum speed limit. From a remarkably simple relation, we concluded that any quantum manybody system whose energy variance scales like $N^{\alpha}$ exhibits the exponential sensitivity to local perturbations. This insight was demonstrated and validated for the trapped Fermi gas, which closely resembles the situation originally studied by Anderson [32]. As a second example, we analyzed the isotropic LMG model interacting with a single qubit impurity, showing that emergence of the orthogonality catastrophe is dependent on the phase the environment is initialized in. Finally, we also proposed a new QSL that relates the work necessarily performed by the local perturbation for the orthogonality catastrophe to appear. In particular, the last two results may justify further study and encourage the development of a comprehensive thermodynamic framework for quantum many-body systems.

T. F. acknowledges support under JSPS KAKENHI18K13507. S. D. acknowledges support from the U.S. NSF under Grant No. CHE-1648973. This research was supported by Grant No. FQXi-RFP-1808 from the Foundational Questions Institute and Fetzer Franklin

*thomas.fogarty@oist.jp

†deffner@umbc.edu

thomas.busch@oist.jp

${ }^{\S}$ steve.campbell@ucd.ie

[1] E. A. Cornell and C. E. Wieman, Nobel Lecture: BoseEinstein condensation in a dilute gas, the first 70 years and some recent experiments, Rev. Mod. Phys. 74, 875 (2002).

[2] W. Ketterle, Nobel lecture: When atoms behave as waves: Bose-Einstein condensation and the atom laser, Rev. Mod. Phys. 74, 1131 (2002).

[3] M.Z. Hasan and C. L. Kane, Colloquium: Topological insulators, Rev. Mod. Phys. 82, 3045 (2010).

[4] A. Bansil, H. Lin, and T. Das, Colloquium: Topological band theory, Rev. Mod. Phys. 88, 021004 (2016).

[5] N. M. R. Peres, Colloquium: The transport properties of graphene: An introduction, Rev. Mod. Phys. 82, 2673 (2010).

[6] N. P. Armitage, E. J. Mele, and A. Vishwanath, Weyl and Dirac semimetals in three-dimensional solids, Rev. Mod. Phys. 90, 015001 (2018).

[7] D. A. Abanin, E. Altman, I. Bloch, and M. Serbyn, Colloquium: Many-body localization, thermalization, and entanglement, Rev. Mod. Phys. 91, 021001 (2019).

[8] S. Deffner and S. Campbell, Quantum Thermodynamics (Morgan \& Claypool Publishers, San Rafael, 2019).

[9] S. Deffner and S. Campbell, Quantum speed limits: From Heisenberg's uncertainty principle to optimal quantum control, J. Phys. A 50, 453001 (2017).

[10] W. Heisenberg, Über den anschaulichen Inhalt der quantentheoretischen Kinematik und Mechanik, Z. Phys. 43, 172 (1927).

[11] L. Mandelstam and I. Tamm, The uncertainty relation between energy and time in nonrelativistic quantum mechanics, J. Phys. 9, 249 (1945).

[12] K. Bhattacharyya, Quantum decay and the MandelstamTamm-energy inequality, J. Phys. A 16, 2993 (1983).

[13] J. Uffink, The rate of evolution of a quantum state, Am. J. Phys. 61, 935 (1993).

[14] N. Margolus and L. B. Levitin, The maximum speed of dynamical evolution, Physica (Amsterdam) 120D, 188 (1998).

[15] V. Giovannetti, S. Lloyd, and L. Maccone, Quantum limits to dynamical evolution, Phys. Rev. A 67, 052109 (2003).

[16] P. Pfeifer, How Fast Can a Quantum State Change With Time?, Phys. Rev. Lett. 70, 3365 (1993).

[17] P. M. Poggi, F. C. Lombardo, and D. A. Wisniacki, Quantum speed limit and optimal evolution time in a two-level system, Europhys. Lett. 104, 40005 (2013).

[18] S. Deffner and E. Lutz, Energy-time uncertainty relation for driven quantum systems, J. Phys. A 46, 335302 (2013). 
[19] G. C. Hegerfeldt, Driving at the Quantum Speed Limit: Optimal Control of a Two-Level System, Phys. Rev. Lett. 111, 260501 (2013).

[20] E. Barnes, Analytically solvable two-level quantum systems and Landau-Zener interferometry, Phys. Rev. A 88, 013818 (2013).

[21] M. Bukov, D. Sels, and A. Polkovnikov, Geometric Speed Limit of Accessible Many-Body State Preparation, Phys. Rev. X 9, 011034 (2019).

[22] N. Il'in and O. Lychkovskiy, Quantum speed limits for adiabatic evolution, Loschmidt echo and beyond, arXiv: 1805.04083

[23] A. del Campo, I. L. Egusquiza, M. B. Plenio, and S. F. Huelga, Quantum Speed Limits in Open System Dynamics, Phys. Rev. Lett. 110, 050403 (2013).

[24] M. M. Taddei, B. M. Escher, L. Davidovich, and R. L. de Matos Filho, Quantum Speed Limit for Physical Processes, Phys. Rev. Lett. 110, 050402 (2013).

[25] S. Deffner and E. Lutz, Quantum Speed Limit for Non-Markovian Dynamics, Phys. Rev. Lett. 111, 010402 (2013).

[26] S. Deffner, Optimal control of a qubit in an optical cavity, J. Phys. B 47, 145502 (2014).

[27] A. D. Cimmarusti, Z. Yan, B. D. Patterson, L. P. Corcos, L. A. Orozco, and S. Deffner, Environment-Assisted SpeedUp of the Field Evolution in Cavity Quantum Electrodynamics, Phys. Rev. Lett. 114, 233602 (2015).

[28] I. Marvian and D. A. Lidar, Quantum Speed Limits for Leakage and Decoherence, Phys. Rev. Lett. 115, 210402 (2015).

[29] D. P. Pires, M. Cianciaruso, L. C. Céleri, G. Adesso, and D. O. Soares-Pinto, Generalized Geometric Quantum Speed Limits, Phys. Rev. X 6, 021031 (2016).

[30] S. Deffner, Geometric quantum speed limits: A case for Wigner phase space, New J. Phys. 19, 103018 (2017).

[31] F. Campaioli, F. A. Pollock, F. C. Binder, and K. Modi, Tightening Quantum Speed Limits for Almost All States, Phys. Rev. Lett. 120, 060409 (2018).

[32] P. W. Anderson, Infrared Catastrophe in Fermi Gases with Local Scattering Potentials, Phys. Rev. Lett. 18, 1049 (1967).

[33] P. Zanardi and N. Paunković, Ground state overlap and quantum phase transitions, Phys. Rev. E 74, 031123 (2006).

[34] C. Lupo and M. Schiró, Transient Loschmidt echo in quenched Ising chains, Phys. Rev. B 94, 014310 (2016).

[35] J. Goold, T. Fogarty, N. Lo Gullo, M. Paternostro, and Th. Busch, Orthogonality catastrophe as a consequence of qubit embedding in an ultracold Fermi gas, Phys. Rev. A 84, 063632 (2011).

[36] A. Sindona, J. Goold, N. L. Gullo, S. Lorenzo, and F. Plastina, Orthogonality Catastrophe and Decoherence in a Trapped-Fermion Environment, Phys. Rev. Lett. 111, 165303 (2013).

[37] S. Campbell, M.-Á. García-March, T. Fogarty, and Th. Busch, Quenching small quantum gases: Genesis of the orthogonality catastrophe, Phys. Rev. A 90, 013617 (2014).

[38] M. Schiró and A. Mitra, Transient Orthogonality Catastrophe in a Time-Dependent Nonequilibrium Environment, Phys. Rev. Lett. 112, 246401 (2014).
[39] F. Cosco, Irreversible work and orthogonality catastrophe in the Aubry-André model, arXiv:1809.01855.

[40] S. I. Mistakidis, G. C. Katsimiga, G. M. Koutentakis, Th. Busch, and P. Schmelcher, Quench Dynamics and Orthogonality Catastrophe of Bose Polarons, Phys. Rev. Lett. 122, 183001 (2019).

[41] W. Münder, A. Weichselbaum, M. Goldstein, Y. Gefen, and J. von Delft, Anderson orthogonality in the dynamics after a local quantum quench, Phys. Rev. B 85, 235104 (2012).

[42] M. Knap, A. Shashi, Y. Nishida, A. Imambekov, D. A. Abanin, and E. Demler, Time-Dependent Impurity in Ultracold Fermions: Orthogonality Catastrophe and Beyond, Phys. Rev. X 2, 041020 (2012).

[43] O. Lychkovskiy, O. Gamayun, and V. Cheianov, Time Scale for Adiabaticity Breakdown in Driven Many-Body Systems and Orthogonality Catastrophe, Phys. Rev. Lett. 119, 200401 (2017).

[44] H. T. Quan, Z. Song, X. F. Liu, P. Zanardi, and C. P. Sun, Decay of Loschmidt Echo Enhanced by Quantum Criticality, Phys. Rev. Lett. 96, 140604 (2006).

[45] A. Goussev, D. Waltner, K. Richter, and R. A. Jalabert, Loschmidt echo for local perturbations: Non-monotonic cross-over from the Fermi-golden-rule to the escape-rate regime, New J. Phys. 10, 093010 (2008).

[46] H. J. Lipkin, N. Meshkov, and A. J. Glick, Validity of manybody approximation methods for a solvable model: (I). Exact solutions and perturbation theory, Nucl. Phys. 62, 188 (1965).

[47] H. T. Quan, Z. D. Wang, and C. P. Sun, Quantum critical dynamics of a qubit coupled to an isotropic LipkinMeshkov-Glick bath, Phys. Rev. A 76, 012104 (2007).

[48] L. Hou, B. Shao, and J. Zou, Quantum speed limit for a central system in Lipkin-Meshkov-Glick bath, Eur. Phys. J. D 70, 35 (2016).

[49] S. Dusuel and J. Vidal, Continuous unitary transformations and finite-size scaling exponents in the Lipkin-MeshkovGlick model, Phys. Rev. B 71, 224420 (2005).

[50] P. Ribeiro, J. Vidal, and R. Mosseri, Thermodynamical Limit of the Lipkin-Meshkov-Glick Model, Phys. Rev. Lett. 99, 050402 (2007).

[51] P. Ribeiro, J. Vidal, and R. Mosseri, Exact spectrum of the Lipkin-Meshkov-Glick model in the thermodynamic limit and finite-size corrections, Phys. Rev. E 78, 021106 (2008).

[52] O. Castaños, R. López-Peña, J. G. Hirsch, and E. López-Moreno, Classical and quantum phase transitions in the Lipkin-Meshkov-Glick model, Phys. Rev. B 74, 104118 (2006).

[53] T. Caneva, R. Fazio, and G. E. Santoro, Adiabatic quantum dynamics of the Lipkin-Meshkov-Glick model, Phys. Rev. B 78, 104426 (2008).

[54] S. Campbell, G. De Chiara, M. Paternostro, G. M. Palma, and R. Fazio, Shortcut to Adiabaticity in the Lipkin-MeshkovGlick Model, Phys. Rev. Lett. 114, 177206 (2015).

[55] S. Campbell, Criticality revealed through quench dynamics in the Lipkin-Meshkov-Glick model, Phys. Rev. B 94, 184403 (2016).

[56] For brevity, in the formulas, we work in units such that $\hbar=1$.

[57] A. del Campo, Long-time behavior of many-particle quantum decay, Phys. Rev. A 84, 012113 (2011). 
[58] M. Pons, D. Sokolovski, and A. del Campo, Fidelity of fermionic-atom number states subjected to tunneling decay, Phys. Rev. A 85, 022107 (2012).

[59] A. del Campo, Exact quantum decay of an interacting manyparticle system: The Calogero-Sutherland model, New J. Phys. 18, 015014 (2016).

[60] R. Jafari and H. Johannesson, Loschmidt Echo Revivals: Critical and Noncritical, Phys. Rev. Lett. 118, 015701 (2017).

[61] R. Jafari and H. Johannesson, Decoherence from spin environments: Loschmidt echo and quasiparticle excitations, Phys. Rev. B 96, 224302 (2017).

[62] R. Jafari and A. Akbari, Gapped quantum criticality gains long-time quantum correlations, Europhys. Lett. 111, 10007 (2015).

[63] R. Jafari, Quench dynamics and ground state fidelity of the one-dimensional extended quantum compass model in a transverse field, J. Phys. A 49, 185004 (2016).

[64] P. Nozières and C. T. De Dominicis, Singularities in the $\mathrm{x}$-ray absorption and emission of metals. III. One-body theory exact solution, Phys. Rev. 178, 1097 (1969).

[65] M. Cetina, M. Jag, R. S. Lous, J. T. M. Walraven, R. Grimm, R. S. Christensen, and G. M. Bruun, Decoherence of Impurities in a Fermi Sea of Ultracold Atoms, Phys. Rev. Lett. 115, 135302 (2015).

[66] M. Cetina et al., Ultrafast many-body interferometry of impurities coupled to a Fermi sea, Science 354, 96 (2016).

[67] W. K. Wootters, Statistical distance and Hilbert space, Phys. Rev. D 23, 357 (1981).
[68] S. Boixo, S. T. Flammia, C. M. Caves, and J. M. Geremia, Generalized Limits for Single-Parameter Quantum Estimation, Phys. Rev. Lett. 98, 090401 (2007).

[69] S. Campbell and S. Deffner, Trade-Off between Speed and Cost in Shortcuts to Adiabaticity, Phys. Rev. Lett. 118, 100601 (2017).

[70] D. Šafránek and S. Deffner, Quantum Zeno effect in correlated qubits, Phys. Rev. A 98, 032308 (2018).

[71] M. A. García-March, T. Fogarty, S. Campbell, T. Busch, and M. Paternostro, Non-equilibrium thermodynamics of harmonically trapped bosons, New J. Phys. 18, 103035 (2016).

[72] K. Funo, J.-N. Zhang, C. Chatou, K. Kim, M. Ueda, and A. del Campo, Universal Work Fluctuations During Shortcuts to Adiabaticity by Counterdiabatic Driving, Phys. Rev. Lett. 118, 100602 (2017).

[73] See Supplemental Material at http://link.aps.org/ supplemental/10.1103/PhysRevLett.124.110601 for the derivation of Eq. (8) and details regarding the energy spectrum and survival probability for a quenched LMG spin bath.

[74] A. Silva, Statistics of the Work Done on a Quantum Critical System by Quenching a Control Parameter, Phys. Rev. Lett. 101, 120603 (2008).

[75] P. Talkner and P. Hänggi, Aspects of quantum work, Phys. Rev. E 93, 022131 (2016).

[76] S. Waldenstrøm and K. Razi Naqvi, The overlap integrals of two harmonic-oscillator wavefunctions: Some remarks on originals and reproductions, Chem. Phys. Lett. 85, 581 (1982). 